\title{
Explanation for the low flux of high energy astrophysical muon neutrinos
}

\author{
Sandip Pakvasa \\ Department of Physics and Astronomy, University of Hawaii, Honolulu, HI 96822 U.S.A. \\ Anjan Joshipura and Subhendra Mohanty \\ Physical Research Laboratory, Ahmedabad, India 380009
}

(Dated: September 19, 2018)

\begin{abstract}
There has been some concern about the unexpected paucity of cosmic high energy muon neutrinos in detectors probing the energy region beyond $1 \mathrm{PeV}$. As a possible solution we consider the possibility that some exotic neutrino property is responsible for reducing the muon neutrino flux at high energies from distant sources; specifically, we consider: (i) neutrino decay and (ii) neutrinos being pseudo-Dirac particles. This would provide a mechanism for the reduction of high energy muon events in the IceCube detector, for example.

PACS numbers:
\end{abstract}

The most recent data from the IceCube collaboration[1] place stringent limits on the muon neutrino flux at high energies from astrophysical sources. The new limits appear to put severe bounds on models of neutrino production in GRB's and AGN's[2]. Similarly, other experiments probing the ultra-high-energy regime, such as ANITA [3] and AUGER [4] have not seen any evidence of long anticipated cosmic neutrinos. It should be noted that very recently there have been re-evaluations of the expected neutrino fluxes from GRB's, especially following the stringent upper limits from IceCube [1]. It has been pointed out [5, 6] that IceCube [1] calculation of the WB neutrino flux from the observed gamma ray flux may have been an overestimation by as much as a factor of 5. So the discrepancy may not be that dire, yet; but the possibility remains that as the bounds get tighter with future observations, the Waxman-Bahcall models [2] will be challenged. In such an eventuality, we would like to offer in this letter the possibility of other causes for the smallness of the muon neutrino flux, which arise from neutrino properties. We note that there are alternative astrophysical models ( see [7, 8] and references therein) which predict a lower neutrino flux compared to the Waxman-Bahcall models [2].

In this note we would like to raise the possibility that these severe bounds are illusory because the small flux may be due to depletion of muon neutrinos which in turn is caused by neutrino properties We consider two possible scenarios. One is that neutrino decay is responsible for depletion of muon-neutrinos and the other is that neutrinos are pseudo-Dirac particles and there is leakage into the sterile components of the pseudo-Dirac particles. Both of these were considered almost ten years ago [9, 10], but the focus then was on the modification of the flavor mix from the canonical 1:1:1 as expected from conventional flavor oscillations with the known neutrino mixings [11].

In the following, we describe both possibilities. To be definite, we are considering neutrino energies in the vicin- ity of order of a $\mathrm{PeV}$, and the distances from the sources of order of hundreds of mega-parsecs. In principle, when the distances become large enough, the cosmological red shift becomes important, and the travel distance $\mathrm{L}$ is limited; these effects were discussed some time ago in ref. 10, 12] and more recently in ref. 13] and ref. [14].

Of course, because of the uncertainty in predicting fluxes, we do not know precisely what amount of depletion is needed. But the scenarios we suggest below can provide a wide range of suppression ranging from none to an order of magnitude.

\section{Neutrino Decay:}

We consider here scenarios with three light neutrinos and assume that the source distances are large enough so that two of the three mass eigenstates, specifically $\nu_{2}$ and $\nu_{3}$ have decayed away completely. If the neutrino masses are quasi-degenerate, that is the masses of $\nu_{2}$ and $\nu_{3}$ are close to that of $\nu_{1}$, then the daughter neutrino, $\nu_{1}$ carries most of the energy of the parent, and so contributes to the flux at that energy; in this case even though the final state is pure $\nu_{1}$, there is not much depletion. So for our purpose here, the preferred mass spectrum is quasihierarchical, namely $m_{2}$ and $m_{3}$ much larger than $m_{1}$; in this case the daughter neutrino energy is much lower than the parent and the final $\nu_{1}$ does not contribute to the flux at that energy and can be counted out. This is discussed in detail in several papers, especially clearly in ref. [15]. This means that the exponential decay factor exp $(-L / \gamma c \tau)$ is negligibly small for them. Since distances to GRB's are of order of 100's of MPc, for energies in the $\mathrm{PeV}$ range, $\frac{L}{\gamma c \tau}=\frac{L}{E}\left(\frac{m c^{2}}{c \tau}\right) \gg 1$ corresponds to $\tau / m<10^{3} \mathrm{sec} / \mathrm{eV}$ where $\tau$ is the rest frame lifetime. A lower bound on the lifetime follows from the BBN (Big Bang Nucleosynthesis). If the standard picture is to remain intact then all three neutrinos must be present and in equilibrium in the BBN era so that the crucial n/p ratio and the nuclear abundances as obtained in standard 
picture remains unaffected. This puts a lower bound of $\frac{\tau}{m} E>1 \mathrm{sec}$ on the neutrino lifetime with $E \sim \mathrm{MeV}$. These considerations restrict the allowed window of lifetime in the range

$$
10^{-6} \text { sec. } / \mathrm{eV} \leq \frac{\tau}{m} \leq 10^{3} \text { sec. } / \mathrm{eV} .
$$

As for the neutrino decay modes, we know the following. The radiative decays such as $\nu_{i} \rightarrow v_{j}+\gamma$ are severely constrained by their contribution to $\nu+e \rightarrow e+\nu^{\prime}$ and from the current bounds on such contributions the radiative decay lifetime must satisfy [16].

$$
\tau_{i} / m_{i}>10^{17} \mathrm{sec} . / \mathrm{eV} .
$$

The three-body invisible decay mode

$$
\nu_{i} \rightarrow \nu_{j}+\nu \bar{\nu}
$$

is constrained by $\mathrm{BBN}$ and the deviation of the invisible width of $\mathrm{Z}$ from the expected value (with three neutrinos) in SM [17]; and is given by

$$
\tau_{i} / m_{i}>10^{28} \mathrm{sec} . / \mathrm{eV}
$$

The kinds of decay models possible are quite restricted. Models where the coupling is chirality conserving (e.g. into a light vector boson or into a scalar boson with a derivative coupling), would by $S U(2)_{L} \times U(1)$ symmetry lead to flavor changing decays of charged leptons at the same strength. The severe bounds on flavor changing decays of $\mu$ and $\tau$ into invisible two body modes lead to limits on lifetimes of $\nu_{2}$ and $\nu_{3}$ of order of $\tau>10^{20} \sec$ [18], and so such decays are ruled out. Hence, the only neutrino decay modes which can be relevant for the short lifetimes of interest here are helicity changing decays into a neutrino and a light boson, as discussed in ref.[9, 16]. The current limits on the lifetimes of the three mass eigenstates are as follows. The most stringent is on that of $\nu_{1}$, from the observation of neutrinos from SN1987A as being about $\tau_{1} / m_{1}>10^{5} \mathrm{~s} / \mathrm{eV}[19]$. The limits on the other two mass eigenstates are: $\tau_{2} / m_{2}>10^{-4} \mathrm{~s} / \mathrm{eV}$ from the solar neutrino observations [15, 20] and $\tau_{3} / \mathrm{m}_{3}>10^{-10} \mathrm{~s} / \mathrm{eV}$ from the atmospheric neutrino observations [21]. Obviously, the limits on the lifetimes of $\nu_{2}$ and $\nu_{3}$ are quite weak.

In the picture adopted here, all the neutrinos originating from GRBs reach the earth as pure $\nu_{1}$ whose flavour content is $e: \mu: \tau=\left|U_{e 1}\right|^{2}:\left|U_{\mu 1}\right|^{2}:\left|U_{\tau 1}\right|^{2}$ as observed long ago [22]. If we insert the current best fit values 23 for the Maki-Nakagawa-Sakata-Pontecorvo (MNSP) 24] neutrino mixing matrix elements, we find that $\left|U_{\mu 1}\right|^{2}$ ranges between 0.1 and 0.3 with a central value of about 0.16 . [The unknown value of the $\mathrm{CP}$ violating phase $\delta$ in the MNSP mixing matrix determines the precise value]. This is a suppression beyond the factor of two due to the standard flavor oscillations. Thus, a suppression of the muon neutrino flux by an order of magnitude is easily achieved. Since the value of $\left|U_{e 1}\right|^{2}$ is between 0.65 and
0.72 , the $\nu_{e}$ flux is not affected much by the decays of $\nu_{2}$ and $\nu_{3}$. We note that the flux ratio of $\nu_{e}$ to $\nu_{\mu}$ is between 2.5 and 8 with a central value of about 4 , depending on the value of the phase $\delta$. We have discussed the most favorable scenario for $\nu_{\mu}$ flux reduction by assuming (i) normal hierarchy, because in the inverted hierarchy the decay of $\nu_{1}$ has strong limits from SN1987a so only $\nu_{2}$ can decay into $\nu_{3}$ but in that case we do not achieve any suppression of $\nu_{\mu}$ and (ii) hierarchial masses, namely $m_{2}, m_{3} \gg m_{1}$; otherwise if the masses are degenerate, the energy of the decaying and daughter neutrino are the same and even though the flavor ratio $\nu_{e} / \nu_{\mu}$ is large there is not much suppression of $\nu_{\mu}$ flux because of enhancement of the $\nu_{1}$ flux from the decay.

The invisible decays $\nu_{2,3} \rightarrow \nu_{1}+J$ arise naturally in Majoron models with $J$ identified with the massless Majoron arising from the spontaneous breaking of total lepton number or some combination of $L_{i}, i=e, \mu, \tau$. These models fall in two main categories: triplet majoron models [25] with a low scale lepton number violation and singlet models [26] with lepton number typically broken at high scale. The former class of models give a large contribution to the invisible decay width of the $Z$ boson and are ruled out. The singlet majoron models are consistent with the $Z$ decay width but mixing of Majorn with the doublet Higgs in this case lead to rapid energy loss from stars through majoron emission. This can be prevented if lepton number breaking occurs at a high scale, (typically $>10^{7} \mathrm{GeV}$ ). It is however possible to consider hybrid models in which Majoron is a combination of the $S U(2)_{L}$ doublet, triplet and singlet. Such models allow low lepton number breaking scale and can be made consistent with the existing experimental constraints [27].

The Majoron couplings to neutrinos is flavour diagonal in simplest triplet model [25] and are nearly so in singlet majoron [26] models. Both these do not allow short neutrino lifetime as required in eq.(1) but such life times can be achieved by allowing majoron to be associated with a spontaneous breaking of some combination of lepton numbers [28] and may also need extension of the simplest model. Denote the coupling relevant to decay as

$$
g_{1 a} \frac{m_{a}}{f} \bar{\nu}_{1} \gamma_{5} \nu_{a} J
$$

where $m_{a}, a=2,3$ is the mass of the decaying neutrino, $f$ is the symmetry breaking scale and $g_{1 a}$ is a model dependent overall coupling. The allowed window for the life time as given in eq.(1) constrains the symmetry breaking scale to lie in the range

$$
8 \mathrm{eV} \leq \frac{f}{g_{1 a}} \leq 0.15 \mathrm{MeV}
$$

for normal hierarchy with $m_{a} \sim 0.05 \mathrm{eV}$. This in particular rules out models with lepton number broken at very high scale but hybrid models [27, 28] with a low $f$ are still allowed.

It has been pointed out 29] that neutrino interactions with a light scalar can make the neutrino fluid 
tightly coupled at the time of photon decoupling (when $T_{\gamma}=0.256 \mathrm{eV}$ ). If neutrinos do not free-stream after photons decouple then they can be a source for photon perturbations which would be observable in CMB anisotropy data. If all neutrinos are assumed to be freesteaming during decoupling then neutrino-Majoron coupling and hence the scale $f$ is $\frac{f}{g_{1 a}}>10^{11} m_{a} \sim 5 \mathrm{GeV}$ which would rule out decay of $\mathrm{PeV}$ neutrinos over cosmological distances of $100 \mathrm{Mpc}$. However it has been shown in [30] that the CMB data does not preclude one or even two neutrino species from being strongly coupled $\left(g_{1 a} m_{a} / f\right)>10^{-7}$ and this still keeps the possibility of UHE neutrino decay viable. The recent Planck data may be able to put stronger constraints on the number of tightly coupled neutrinos at decoupling and rule out the possibility of UHE neutrino invisible decays 31.

Amongst other consequences, the neutrino counting in early universe is modified from a count of 3 to $3+4 / 7$ due to the extra bosonic degree of freedom. This is consistent with most recent cosmological bounds 32 .

The bottom line is that if neutrinos decay, substantial reduction in $\nu_{\mu}$ fluxes is possible, and consistent with $\nu_{1}$ being the lightest mass eigenstate.

\section{Pseudo-Dirac Neutrinos:}

If each of the three neutrino mass eigenstates is actually a doublet with very small mass difference (smaller than $10^{-6} \mathrm{eV}$ ), then there are no current experiments that could have detected this. Such a possibility was raised long ago [33]. It turns out that the only way to detect such small mass differences in the range $\left(10^{-12} \mathrm{eV}^{2}>\right.$ $\delta m^{2}>10^{-18} \mathrm{eV}^{2}$ ) is by measuring flavor mixes of the high energy neutrinos from cosmic sources.

Let $\left(\nu_{1}^{+}, \nu_{2}^{+}, \nu_{3}^{+} ; \nu_{1}^{-} \nu_{2}^{-}, \nu_{3}^{-}\right.$denote the six mass eigenstates where $\nu^{+}$and $\nu^{-}$are a nearly degenerate pair. A $6 \times 6$ mixing matrix rotates the mass basis into the flavor basis $\left(\nu_{e}, \nu_{\mu}, \nu_{\tau} ; \nu_{e}^{\prime}, \nu_{\mu}^{\prime}, \nu_{\tau}^{\prime}\right)$. In general, for six Majorana neutrino, there would be fifteen rotation angles and fifteen phases. However, for pseudo-Dirac neutrinos, Kobayashi and Lim [34] have given an elegant proof that the 6x6 matrix $V_{K L}$ takes the very simple form

$$
V_{K L}=\left(\begin{array}{cc}
U & 0 \\
0 & U_{R}
\end{array}\right) \cdot\left(\begin{array}{cc}
V_{1} & i V_{1} \\
V_{2} & -i V_{2}
\end{array}\right)
$$

where the $3 \times 3$ matrix $U$ is just the usual mixing matrix; the $3 \times 3$ matrix $U_{R}$ is an unknown unitary matrix and $V_{1}$ and $V_{2}$ are the diagonal matrices $V_{1}=\operatorname{diag}(1,1,1) / \sqrt{2}$, and $V_{2}=\operatorname{diag}\left(e^{-i \phi 1}, e^{-i \phi 2}, e^{-i \phi 3}\right) / \sqrt{2}$ with the $\phi_{i}$ being arbitrary phases. A very similar mass spectrum can be produced in the mirror model [35].

As a result, the three active neutrino states are described in terms of the six mass eigenstates as:

$$
\nu_{\alpha L}=U_{\alpha j} \frac{1}{\sqrt{2}}\left(\nu_{j}^{+}+i \nu_{j}^{-}\right) .
$$

The nontrivial matrices $U_{R}$ and $V_{2}$ are not accessible to active flavor measurements. The flavor conversion probability can thus be expressed as

$$
P_{\alpha \beta}=\frac{1}{4}\left|\sum_{j=1}^{3} U_{\alpha j}\left\{e^{i\left(m_{j}^{+}\right)^{2} l / 2 E}+e^{i\left(m_{j}^{-}\right)^{2} l / 2 E}\right\} U_{\beta j}^{*}\right|^{2}
$$

In the description of the three active neutrinos, the only new parameters are the three pseudo-Dirac mass differences, $\delta m_{j}^{2}=\left(m_{j}^{+}\right)^{2}-\left(m_{j}^{-}\right)^{2}$. In the limit that they are negligible, the oscillation formulas reduce to the standard ones and there is no way to discern the pseudoDirac nature of the neutrinos.

Incidentally, the effective mass for neutrino-less double beta decay is given by

$$
\langle m\rangle_{\text {eff }}=\frac{1}{2} \sum_{j} U_{e j}^{2}\left(m_{j}^{+}-m_{j}^{-}\right)=\frac{1}{2} \sum_{j} U_{e j}^{2} \frac{\delta m_{j}^{2}}{2 m_{j}}
$$

The value of this effective mass is smaller than $10^{-4} \mathrm{eV}$ for inverted hierarchy and smaller for normal hierarchy and renders neutrinoless double beta decay unobservable.

When $\mathrm{L} / \mathrm{E}$ becomes large enough, flavor fluxes will deviate from the canonical value of $1 / 3$ by [10]

$$
\delta P_{\beta}=\frac{1}{3}\left[\left|U_{\beta 1}\right|^{2} \chi_{1}+\left|U_{\beta 2}\right|^{2} \chi_{2}+\left|U_{\beta 3}\right|^{2} \chi_{3}\right]
$$

where $\chi_{i}=\sin ^{2}\left(\delta m_{i}^{2} L / 4 E\right)$

We assume that for the neutrinos from distant sources arriving in IceCube, $\chi_{1} \approx 0$ but $\chi_{2}=\chi_{3} \approx 1 / 2$; i.e. $\delta m_{1}^{2}<<\delta m_{2}^{2}$ and $\delta m_{3}^{2}$. For example, if $\delta m_{1}^{2}<<$ $10^{-17} \mathrm{eV}^{2}$ and $\delta m_{2}^{2}, \delta m_{3}^{2} \sim{ }^{\sim} 0^{-15} \mathrm{eV}^{2}$ then the condition for $\chi_{1} \approx 0$ and $\chi_{2}=\chi_{3} \approx \frac{1}{2}$ for GRB neutrinos is satisfied.

The deviation from $1 / 3$ for $\nu_{\mu}^{\prime} s$ is given by

$$
\delta P_{\mu}=-\frac{1}{3}\left[\frac{1}{2}\left(\left|U_{\mu 2}\right|^{2}+\left|U_{\mu 3}\right|^{2}\right)\right]
$$

Using the current best values for the mixing parameters [23], this can be very close $1 / 6$, thus giving an extra reduction by a factor of 2 for the flux of $\nu_{\mu}^{\prime} s$. In a model for pseudo-Dirac neutrinos via Mirror-world, a further suppression by a factor $1 / 2$ obtains resulting in a net suppression by a factor of $1 / 4$ 36. Furthermore, the shift in $P_{e}$ from the value $1 / 3$ is about 0.8 , and so the ratio $\nu_{e} / \nu_{\mu}$ is about 3 .

This is a very different physics possibility from the decay case but also gives rise to low fluxes of $\nu_{\mu} s$ consistent with the lack of observation in IceCube.

To summarize, we raise two rather different possibilities of neutrino properties which can account for the low fluxes of $\nu_{\mu}^{\prime} s$ at high energies, and give rather large values for the ratio of $\nu_{e}$ to $\nu_{\mu}$ fluxes. The two can be distinguished in several ways. The decay changes the primordial neutrino counting from 3 to $3+4 / 7$, and the pseudoDirac neutrinos make the neutrinoless double beta decay unobservable. The flavour ratios $\nu_{e} / \nu_{\mu}$ is another 
clear indicator of the mechanism responsible; in the decay case it may vary between 2.5 and 8 , whereas is 3 for the pseudo-Dirac case. Only further experimental data can confirm or rule out these speculations. Since the scenarios considered here do not suppress the electron neutrino flux, we have no problem with the $\mathrm{PeV}$ shower events reported by IceCube at the Neutrino 2012 meeting in June 37].

If $\nu_{\mu}$ events in $\mathrm{PeV}$ energy range are seen in Icecube, the drastic explanation offered here becomes unnecessary. In that case, the observed flavor ratios can be used to constrain parameters of models such as the ones discussed here as has been discussed before [38].

Most of the material in this letter was presented by one of the authors(S.P.) at the "What's nu-Invisibles" workshop at GGI, Florence in June 2012 and at the CETUP workshop, Lead, S. D. in July 2012.

\section{Acknowledgments:}

We thank John Learned for discussions and a careful reading of the manuscript and we thank Danny Marfatia for useful discussions. This work is supported in part by US DOE Grant DE-FG02-04ER41291 and by the Indo-U.S. Science and Technology Forum under Grant IUSSTF/JC/Physics Beyond Standard Model/232010/2010-2011. We also thank the Center for Theoretical Underground Physics and Related Areas (CETUP* 2012) for its support and hospitality. ASJ thanks the Department of Science and Technology, Government of India for support under the J. C. Bose National Fellowship programme, grant no. SR/S2/JCB-31/2010.
[1] R. Abbasi et al., [IceCube Collaboration], Nature 484, 351 (2012).

[2] E. Waxman and J. Bahcall, Phys. Rev. Lett. 78, 2292 (1997); J.P. Rachen and P. Meszaros, arXiv: astro-ph/9811266 D. Guetta et al., Astropart Phys. 20, 429 (2004); M. Ahlers, M.C. Gonzalez-Garcia and F. Halzen, Astroparticle Phys. 35, 87 (2011).

[3] A. G. Vieregg et al., Astrophys. J. 736, 50 (2011).

[4] Pierre Auger Collaboration (P. Abreu et al.), Astrophys. J. 755, L4 (2012).

[5] Z. Li, Phys. Rev. D 85, 027301 (2012).

[6] S. Hummer, P. Baerwald and W. Winter, Phys. Rev. Lett. 108, 231101 (2012).

[7] S. Gao, K. Asano, P. Meszaros and , JCAP 1211, 058 (2012).

[8] P. Baerwald, M. Bustamante and W. Winter , arXiv:1301.6163 [astro-ph.HE].

[9] J.F. Beacom et al, Phys. Rev. Lett. 90, 181301 (2003); J.F. Beacom et al, Phys. Rev. D69, 017303 (2004).

[10] J.F. Beacom et al., Phys. Rev. Lett. 92, 011101 (2004); R. M. Crocker, F. Melia and R. R. Volkas, Astrophys. J. Suppl. 130, 330 (2000); Astrophys. J. Suppl. 141, 147(2002).

[11] J.G. Learned and S. Pakvasa, Astropart. Phys. J. 3, 267 (1995).

[12] T. J. Weiler, W. A. Simmons, S. Pakvasa and J. G. Learned, hep-ph/9411432 T.J. Weiler and D. Wagner, Mod. Phys. Lett. A12, 2497 (1997).

[13] P. Baerwald, M. Bustamante and W. Winter, JCAP 1210, 020 (2012).

[14] A. Esmaili and Y. Farzan, JCAP 1212, 014 (2012).

[15] J. F. Beacom and N. F. Bell, Phys. Rev. D 65, 113009 (2002).

[16] S. Pakvasa, "Do neutrinos decay?," AIP Conf. Proc. 542, 99 (2000). hep-ph/0004077.

[17] M.S. Bilenky and A. Santamaria, arXiv:hep-ph/9908272

[18] J. Beringer et al. (Particle Data Group), Phys. Rev. D86, 010001 (2012); A. Jodidio et al, Phys. Rev. D34, 1967(1986).

[19] The Kamiokande Collaboration (K.Hirata et al). Phys. Rev. Lett. 58, 1490 (1987); The IMB Collaboration (R.M.
Bionta et al.) ibid 58, 1494 (1987).

[20] A. Joshipura, E. Masso and S. Mohanty, Phys. Rev. D66, 13008(2002); The Kamland Collaboration (K. Eguchi et al.) Phys. Rev. Lett. 92, 071301 (2004).

[21] M. C. Gonzalez-Garcia and M. Maltoni, Phys. Lett.B, 663, 405(2008).

[22] S. Pakvasa, Lett. Nuov. Cim 31, 497 (1981).

[23] T. Schwetz, et al, Review of Global fits presented at "What's $\nu$-Invisibles" GGI Workshop, Florence, June 2012; M. C. Gonzalez-Garcia, M. Maltoni, J. Salvado and T. Schwetz, JHEP 1212, 123 (2012); G. L. Fogli, E. Lisi, A. Marrone, D. Montanino, A. Palazzo and A. M. Rotunno, Phys. Rev. D 86, 013012 (2012).

[24] B. Pontecorvo, Zh. Eksp. Teor. Fiz. 33, 549 (1957); B. Pontecorvo, Zh. Eksp. Teor. Fiz. 34, 247 (1958);V. N. Gribov and B. Pontecorvo, Phys. Lett. B 28, 493 (1969); Z. Maki, N. Nakagawa and S. Sakata, Prog. Theor. Phys 28, 870 (1962).

[25] G. B. Gelmini and M. Roncadelli, Phys. Lett. B 99, 411 (1981).

[26] Y. Chikashige, R. N. Mohapatra and R. D. Peccei, Phys. Rev. Lett. 45, 1926 (1980);

[27] A. S. Joshipura, Int. J. Mod. Phys. A 7, 2021 (1992);K. Choi and A. Santamaria, Phys. Lett. B 267, 504 (1991).

[28] J. W. F. Valle, Phys. Lett. B 131, 87 (1983); G. B. Gelmini and J. W. F. Valle, Phys. Lett. B 142, 181 (1984);A. S. Joshipura and S. D. Rindani, Phys. Rev. D 46, 3000 (1992); A. Acker, A. Joshipura and S. Pakvasa, Phys. Lett. B 285, 371 (1992).

[29] S. Hannestad and G. Raffelt, Phys. Rev. D 72, 103514 (2005).

[30] N. F. Bell, E. Pierpaoli and K. Sigurdson, Phys. Rev. D 73, 063523 (2006).

[31] A. Friedland, K. M. Zurek and S. Bashinsky, arXiv:0704.3271 [astro-ph].

[32] WMAP Collaboration (E. Komatsu et al.), Astrophys. J. Suppl. 192, 18 (2011). R. Keisler et al., Astrophys. J. 743, 28(2011); G. Hinshaw et al., Astrophys. J. Supp. ser. (in press), arXiv:1212.5226.

[33] L. Wolfenstein, Nucl. Phys. B186, 147 (1981); S.T. Petcov, Phys. Lett. B 110, 245 (1982); S.M. Bilensky and 
B. Pontecorvo, Sov. J. Nucl. Phys. 38, 248 (1983).

[34] M. Kobayashi and C.S. Lim, Phys. Rev. D64, 013003 (2001).

[35] See for example:R. Foot, H. Lew and R.Volkas, Phys. Lett. B272, 67(1991), Mod. Phys. Lett. A7, 2567(1992); R. Foot and R. Volkas, Phys. Rev. D52, 6505(1995), V. Berezensky, M. Narayan and F. Vissani, Nucl. Phys. B658(2003).

[36] A. Joshipura, S. Mohanty and S. Pakvasa, in preparation.
[37] A. Ishihara, "Icecube:Ultra-High Energy Neutrinos" Talk at Neutrino 2012, Kyoto Japan, June 2012; slides available at http://neu2012.kek.jp/index.htm

[38] S. Pakvasa, Nuclear Physics B (Proc. Suppl.) 137, 295 (2004), Proc. of the Fujihara Seminar on Neutrino Mass and See-Saw Mehcanism, KEK, Japan, 23-25 Feb. 2004, Ed. by K. Nakamura, Y. Okada and T. Ishida; hep-ph/0412371 